\title{
ESTUDO DA PRECIPITAÇÃO EFETIVA PARA O MUNICÍPIO DE LAVRAS, MG
}

\author{
Silvio César Sampaioํㅜ, Marcus Metri Corrêa ${ }^{2}$, Márcio Antônio Vilas Bôas ${ }^{3}$ \& \\ Luiz Fernando Coutinho de Oliveira ${ }^{4}$
}

\begin{abstract}
RESUMO
A determinação da precipitação efetiva apresenta grande relevância no estudo da precipitação com fins de aplicação na agricultura, visto que esta parcela da precipitação é realmente aquela que estará contribuindo com a água disponível do solo. Em conseqüência, sua quantificação é de grande utilidade nas irrigações suplementares, levando o irrigante a alcançar expressiva economia na condução da irrigação. O objetivo deste trabalho foi realizar um estudo de chuva efetiva mensal, utilizando-se as precipitações prováveis mensais estimadas por meio da distribuição Log-Normal, com dados observados junto à estação climatológica da Universidade Federal de Lavras, no período de 1914 a 1991, para o município de Lavras, MG. Foram utilizados os seguintes métodos para se estimar a precipitação efetiva: percentagem fixa (PF), fórmula empírica (AGLW/FAO) e o do Soil Conservation Service Method (USDA). Considerando-se o nível de probabilidade de $75 \%$, foram encontrados valores de precipitação efetiva anual de 125,4,313,6, 501,8,330,6 e 485,0 mm para os métodos $P F 20,50$ e $80 \%$, FAO e USDA, respectivamente. Para o nível de probabilidade de $90 \%$, encontraram-se os menores totais anuais de precipitação efetiva, sendo: 79,5, 198,8, 318,1, 188,8 e 327,8 mm para os métodos PF 20, 50 e $80 \%$, FAO e USDA, respectivamente.
\end{abstract}

Palavras-chave: hidrologia, chuva efetiva, distribuição log-normal

\section{EFFECTIVE RAINFALL STUDY IN THE MUNICIPALITY OF LAVRAS, MG}

\begin{abstract}
This paper presents a study about the monthly effective rainfall, using the probable precipitation which is calculated by the Log-normal distribution, for the levels of 75 and $90 \%$ of probability, using daily precipitation data from the period of 1914 to 1991, in the region of Lavras - Minas Gerais - Brazil. The data was obtained from the climatologic station of the Universidade Federal de Lavras, employing three methods to calculate the effective rainfall: fixed percentage (PF) (20, 50 and $80 \%)$, dependable rain (FAO/AGLW) and Soil Conservation Service Method (USDA). The results for the level of $75 \%$ of probability rendered an annual effective rainfall of 125.4, 313.6, 501.8, 330.6 and $485.0 \mathrm{~mm}$ for the methods: PF 20, 50 and $80 \%$, FAO and USDA, respectively. For the level of $90 \%$ of probability, the annual effective rainfall found was of 79.5, 198.8, 318.1, 188.8 and $327.8 \mathrm{~mm}$ for the methods: PF 20, 50 and $80 \%$, FAO and USDA, respectively.
\end{abstract}

Key words: hydrology, effective rainfall, log-normal distribution

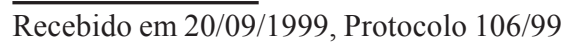

${ }^{1}$ Professor Adjunto da UNIOESTE/CASCAVEL. Rua Universitária, 2069. CEP 85814 - 110, Cascavél, PR. Fone: (0xx45) 225 2100, Ramal: 234.E-mail: ssampaio@unioeste.br

${ }^{2}$ Professor Assistente da UNIOESTE/CASCAVEL. Rua Universitária, 2069. CEP 85814 - 110, Cascavél, PR. Fone: (0xx45) 225 2100, Ramal:234.E-mail:mcorrea@unioeste.br

${ }^{3}$ Professor Adjunto da UNIOESTE/CASCAVEL. Rua Universitária, 2069. CEP 85814 - 110, Cascavél, PR. Fone: (0xx45) 225 2100, Ramal:234.E-mail:vilasma@unioeste.br

${ }^{4}$ Professor Adjunto da Escola de Agronomia da UFG, CP 131, CEP 74001 - 970, Goiânia, GO. E-mail: 1fco@agro.ufg.br 


\section{INTRODUÇÃO}

No Brasil, os projetos de irrigação são geralmente dimensionados em termos de irrigação total, visando cobrir as necessidades hídricas da planta, não se considerando a contribuição da precipitação. Nota-se que nos projetos se utiliza a necessidade de água máxima da cultura para seu dimensionamento e, para fins de manejo, a tendência atual é viabilizar o aproveitamento das precipitações, com a inclusão da chuva efetiva como água disponível às plantas reduzindo, assim, os custos de operação e manutenção do sistema.

Quando a quantidade de irrigação necessária for superestimada, as conseqüências são sistemas super dimensionados, o que, de acordo com Bernardo (1989) encarece o custo de irrigação por unidade de área e leva à aplicação excessiva de água.

A falta de informações climáticas, análises das características da distribuição da precipitação atmosférica e de técnicas baseadas em critérios probabilísticos, que geralmente envolvem riscos, são algumas razões que levam o técnico a utilizar e projetar o sistema para irrigação total. No entanto, Doorembos \& Pruitt (1977), Silva et al. (1988) e Bernardo (1989) recomendam dimensionar o projeto de irrigação levando-se em consideração a precipitação provável com certo nível de probabilidade. Neste sentido, autores como Bernardo et al. (1978), Frizone (1979), Assis (1993) e Sampaio et al. (1999) realizaram vários trabalhos determinando a precipitação provável em períodos semanais, decendiais e mensais, em algumas regiões do Brasil.

Conforme Dastane (1974) apesar de haver um grande volume de dados sobre o tempo, não se conhece o necessário sobre as precipitações. Alguns conceitos simples são confundidos pelos técnicos da área, como o conceito de precipitação efetiva que, no seu sentido mais amplo, significa a precipitação útil ou utilizável; entretanto, este conceito varia bastante, de acordo com a área de interesse. No abastecimento, a água de real interesse é aquela que entra no reservatório e na geração de energia, é interessante a quantidade que escoa superficialmente.

$\mathrm{Na}$ área de produção agrícola, a precipitação efetiva foi definida primeiramente por Hayes \& Buell, citados por Dastane (1974) como sendo a precipitação total menos o escoamento e evaporação; depois, Hershfield (1964) definiu que esta seria a diferença entre a precipitação total menos as perdas por escoamento superficial e percolação profunda. Dastane (1974) cita uma definição mais moderna que aponta as necessidades de água no preparo do solo e lixiviação de sais sendo, deste modo, a precipitação total menos as perdas por escoamento superficial e percolação profunda e a variação de umidade no solo.

Vários fatores influem na porção efetiva da precipitação total, os quais podem atuar isoladamente ou interagindo com outros. Qualquer fator que afete a infiltração, o escoamento superficial ou a evapotranspiração, tem influência no valor da precipitação efetiva.
Dastane (1974) descreve, ainda, vários métodos empíricos de medição e cálculo de precipitação efetiva, entre eles, a equação de Renfro, o método de US Bureau of Reclamation e outros métodos diretos.

No programa CROPWAT, desenvolvido por Smith (1992) o cálculo das necessidades de irrigação é efetuado pela diferença entre a evapotranspiração máxima da cultura e a precipitação efetiva, com base em dados mensais, utilizando alguns métodos empíricos na determinação da precipitação efetiva. Sanchez (1972) e Louzada et al. (1991) afirmam que esta metodologia possui algumas limitações como variação na distribuição de chuvas e umidade do solo no momento da precipitação. Bernardo (1989) também discorda de Smith (1992) quando afirma que o ideal para países tropicais é totalizar os dados em períodos inferiores ao mensal.

É importante ressaltar, no entanto, que os métodos empíricos não podem ser aqui simplesmente descartados, uma vez que qualquer informação científica referente à precipitação efetiva deve ser utilizada pelo técnico, como ponto de partida de um projeto hidroagrícola econômico e funcional, lembrando ainda que esses métodos são geralmente os mais utilizados, por sua simplicidade de aplicação.

O objetivo deste trabalho foi estimar as precipitações efetivas mensais para o município de Lavras, MG, fazendo-se uso das precipitações prováveis estimadas por Sampaio et al. (1999) no período 78 anos, utilizando-se a metodologia sugerida por Smith (1992).

\section{MATERIAL E MÉTODOS}

O trabalho foi desenvolvido na região de Lavras, Minas Gerais, geograficamente definida pelas coordenadas de $21^{\circ} 14^{\prime}$ de latitude sul, $45^{\circ}$ de longitude oeste de Greenwich e $900 \mathrm{~m}$ de altitude. O clima, que possui duas estações definidas, seca de abril a setembro e chuvosa de outubro a março, é classificado como Cwb, segundo a classificação de Köppen.

O estudo foi realizado a partir das estimativas mensais de chuva provável para a região, por Sampaio et al. (1999) cujos dados aqui utilizados compreenderam o período de 1914 a 1991, resultando em 78 anos de observação. As lâminas diárias foram totalizadas em período de 30 dias e avaliadas com níveis de probabilidade de ocorrência de 75 e $90 \%$. Ressalta-se que, considerando-se a análise econômica, o valor de $75 \%$ é sugerido por Bernardo (1989) e Chow (1964) para estudos com fins agrícolas. Utilizou-se a distribuição Log-N, sendo esta a própria distribuição da curva de Gauss, na estimativa da chuva provável. Os resultados desta estimativa podem ser observados na Tabela 1.

Tabela 1. Precipitações prováveis $(\mathrm{mm})$ na região de Lavras, $\mathrm{MG}$, para o intervalo de agrupamento de 30 dias, com probabilidade de 75 e $90 \%$, referentes ao período de 1914 a 1991

\begin{tabular}{|c|c|c|c|c|c|c|c|c|c|c|c|c|c|}
\hline Prob. & Jan & $\mathrm{Fev}$ & Mar & Abr & Mai & Jun & Jul & Ago & Set & Out & Nov & Dez & Total \\
\hline \multicolumn{14}{|c|}{$\mathrm{mm}$} \\
\hline 75 & 81,3 & 59,6 & 69,9 & 16,3 & 4,4 & 1,8 & 0,5 & 0,6 & 9,7 & 68,2 & 130,5 & 184,4 & 702,2 \\
\hline 90 & 36,3 & 26,7 & 37,8 & 7,2 & 1,4 & 0,5 & 0,1 & 0,1 & 3,5 & 44,4 & 101,0 & 138,6 & 487,6 \\
\hline
\end{tabular}


A partir desses dados, realizou-se a estimativa da chuva efetiva para a região de Lavras, $\mathrm{MG}$; esta chuva efetiva foi obtida a partir dos métodos: percentagem fixa (PF) (20, 50 e $80 \%)$, fórmula empírica (AGLW/FAO) e do Soil Conservation Service Method (USDA) fazendo-se uso do programa aplicativo CROPWAT, desenvolvido pela FAO (Smith, 1992).

O método da percentagem fixa é definido como a precipitação efetiva com uma probabilidade de excedência respectiva à percentagem aplicada; assim, 20\% de probabilidade de excedência representam um ano considerado "úmido"; $50 \%$ se referem a um ano "normal", aproximando-se de uma média; já $80 \%$ representam um ano "seco", o que equivale a se dizer, então, que quanto mais úmido o ano, menor a porcentagem da chuva que fica retida no solo disponível às plantas.

O método da AGLW/FAO foi desenvolvido em climas árido e sub-úmido estimando-se perdas por escoamento superficial e percolação. As fórmulas empíricas obtidas foram:

$$
\begin{aligned}
& \mathrm{P}_{\text {efetiva }}=0,6 \mathrm{P}_{\text {Total }}-10 \mathrm{P}_{\text {Total }}<70 \mathrm{~mm} \\
& \mathrm{P}_{\text {efetiva }}=0,6 \mathrm{P}_{\text {Total }}-10 \mathrm{P}_{\text {Total }}>70 \mathrm{~mm}
\end{aligned}
$$

O método USDA foi desenvolvido por meio de balanços hídricos relacionando-se a entrada da precipitação com as saídas, por escoamento superficial e percolação, bem como a água retida no zona radicular, para várias culturas. Os pesquisadores chegaram às seguintes fórmulas:

$$
\begin{aligned}
& \mathrm{P}_{\text {efetiva }}=\frac{\mathrm{P}_{\text {Total }}(125-0,2) \mathrm{P}_{\text {Total }}}{125}<250 \mathrm{~mm} \\
& \mathrm{P}_{\text {efetiva }}=125+0,1 \mathrm{P}_{\text {Total }}>250 \mathrm{~mm}
\end{aligned}
$$

\section{RESULTADOS E DISCUSSÃO}

Os resultados de chuva efetiva para a região estudada encontram-se nas Tabelas 2 e 3. Observa-se que, para o nível de probabilidade de $75 \%$, foram encontrados maiores valores de precipitação efetiva, uma vez que a mesma está diretamente relacionada com a precipitação provável.

Verifica-se que os métodos FAO e USDA possuem valores relativamente próximo aos estimados pelo método PF 50 e $80 \%$, respectivamente mostrando, assim, que a região de Lavras, $\mathrm{MG}$, é classificada entre um clima "normal" e "seco", o que é observado na prática, pois em alguns meses do ano faz-se necessário o uso da irrigação. Este fato poderá ser mais relevante, caso se leve em consideração o relevo da região, já que é bastante irregular, porém nos meses de maior precipitação (novembro e dezembro) o método $\mathrm{PF} 80 \%$ proporciona uma precipitação efetiva maior que o método do USDA, ocorrendo o inverso para os meses de menor precipitação, ou seja, de maio a outubro.

Comparando-se os métodos FAO e USDA, verifica-se que, a medida em que a precipitação provável diminuiu, a precipitação efetiva estimada pelo método da FAO é menor que aquela estimada pelo método USDA.

Os valores de precipitação efetiva a nível de probabilidade de $90 \%$ podem ser utilizados em projetos de irrigação, onde a cultura possui maior sensibilidade ao estresse hídrico, ou naqueles de maior valor econômico; já para outras culturas pode ser utilizado o nível de probabilidade de $75 \%$, seguindo-se recomendações de Doorenbos \& Pruitt (1977), Silva et al. (1988)

\begin{tabular}{|c|c|c|c|c|c|c|c|c|c|c|c|c|}
\hline Jan & $\mathrm{Fev}$ & Mar & Abr & Mai & Jun & Jul & Ago & Set & Out & Nov & Dez & Total \\
\hline \multicolumn{13}{|c|}{ Percentagem Fixa 20\% } \\
\hline 16,3 & 11,9 & 14,0 & 3,3 & 0,9 & 0,4 & 0,1 & 0,1 & 1,9 & 13,6 & 26,1 & 36,9 & 125,4 \\
\hline \multicolumn{13}{|c|}{ Percentagem Fixa 50\% } \\
\hline 40,7 & 29,8 & 35,0 & 8,1 & 2,2 & 0,9 & 0,3 & 0,3 & 4,9 & 34,1 & 65,3 & 92,2 & 313,6 \\
\hline \multicolumn{13}{|c|}{ Percentagem Fixa $80 \%$} \\
\hline 65,0 & 47,7 & 55,9 & 13,0 & 3,5 & 1,4 & 0,4 & 0,5 & 7,8 & 54,6 & 104,4 & 147,5 & 501,8 \\
\hline \multicolumn{13}{|c|}{ Fórmula Empírica (AGLW/FAO) } \\
\hline 40,0 & 25,8 & 31,9 & 0,0 & 0,0 & 0,0 & 0,0 & 0,0 & 0,0 & 30,9 & 79,4 & 122,5 & 330,6 \\
\hline \multicolumn{13}{|c|}{ Soil Conservation Service Method (USDA) } \\
\hline 68,1 & 52,5 & 60,1 & 15,8 & 4,4 & 1,8 & 0,5 & 0,6 & 9,5 & 58,9 & 96,4 & 116,4 & 485,0 \\
\hline
\end{tabular}
e Bernardo (1989).

Tabela 2. Precipitações efetivas ( $\mathrm{mm}$ ) na região de Lavras, MG, para o intervalo de agrupamento de 30 dias, com probabilidade de $75 \%$, estimadas por equações empíricas

\begin{tabular}{|c|c|c|c|c|c|c|c|c|c|c|c|c|}
\hline Jan & $\mathrm{Fev}$ & Mar & Abr & Mai & Jun & Jul & Ago & Set & Out & Nov & $\overline{\text { Dez }}$ & Total \\
\hline \multicolumn{13}{|c|}{ Percentagem Fixa $20 \%$} \\
\hline 7,3 & 5,3 & 7,6 & 1,4 & 0,3 & 0,1 & 0,0 & 0,0 & 0,7 & 8,9 & 20,2 & 27,7 & 79,5 \\
\hline \multicolumn{13}{|c|}{ Percentagem Fixa 50\% } \\
\hline 18,2 & 13,4 & 18,9 & 3,6 & 0,7 & 0,3 & 0,1 & 0,1 & 1,8 & 22,2 & 50,5 & 69,3 & 198,8 \\
\hline \multicolumn{13}{|c|}{ Percentagem Fixa $80 \%$} \\
\hline 29,0 & 21,4 & 30,2 & 5,8 & 1,1 & 0,4 & 0,1 & 0,1 & 2,8 & 35,5 & 80,8 & 110,9 & 318,1 \\
\hline \multicolumn{13}{|c|}{ Fórmula Empírica (AGLW/FAO) } \\
\hline 11,8 & 6,0 & 12,7 & 0,0 & 0,0 & 0,0 & 0,0 & 0,0 & 0,0 & 16,6 & 55,8 & 85,9 & 188,8 \\
\hline \multicolumn{13}{|c|}{ Soil Conservation Service Method (USDA) } \\
\hline 33,7 & 25,3 & 34,9 & 7,1 & 1,4 & 0,5 & 0,1 & 0,1 & 3,5 & 40,5 & 80,6 & 100,2 & 327,8 \\
\hline
\end{tabular}

Tabela 3. Precipitações efetivas ( $\mathrm{mm}$ ) na região de Lavras, MG, para o intervalo de agrupamento de 30 dias, com probabilidade de $90 \%$, estimadas por equações empíricas 
Nesse nível de probabilidade para solos com baixa capacidade de infiltração e de armazenamento e de topografia com declive acentuado, recomendam-se os valores obtidos pelo método de PF 20\%; para solos de textura média recomendam-se os valores obtidos pelos métodos PF 50\% e AGLW/FAO. Para solos com alta capacidade de armazenamento, vegetado e com técnicas conservacionistas, pode-se utilizar os resultados obtidos pelo método PF $80 \%$ e USDA. Essas afirmações servem de referência ou de ponto de partida para os técnicos dessa região, no dimensionamento e manejo de projetos de irrigação, pois o ideal seria confrontar esses resultados com algumas características de solos da região, como velocidade de infiltração, textura, capacidade de armazenamento, topografia etc, conforme as propostas de Sanchez (1972) e Louzada et al. (1991).

\section{CONCLUSÕES}

1. Para o nível de probabilidade de $75 \%$, os totais anuais da precipitação efetiva são: 125,4,313,6, 501,8, 330,6 e 485,0 mm; pelos métodos PF, 20, 50 e $80 \%$, FAO e USDA, respectivamente.

2. Para o nível de probabilidade de $90 \%$, os totais anuais da precipitação efetiva são: 79,$5 ; 198,8 ; 318,1 ; 188,8$ e 327,8 mm; pelos métodos PF, 20, 50 e $80 \%$, FAO e USDA, respectivamente.

3. Os valores mensais obtidos pelos métodos FAO e USDA se aproximam dos estimados pelo método PF 50 e $80 \%$, respectivamente, para os períodos intermediários entre as estações de seca e de chuvas, principalmente.

\section{REFERÊNCIAS BIBLIOGRÁFICAS}

ASSIS, F.N. Ajuste da função Gama aos totais semanais de chuva de Pelotas, RS. Revista Brasileira de Agrometeorologia, Santa Maria, v.1, p.131-136. 1993.

BERNARDO, S. Manual de irrigação. $5^{\text {a }}$ Ed. Viçosa, UFV. Imprensa Universitária. 1989. 586p.
BERNARDO, S.; HILL, R.W. Um modelo para determinação de irrigação suplementar. Revista Ceres, Viçosa, v.25, n.140, p.245-262. 1978.

CHOW, Ven Te. Handbook of applied hydrology. New York: McGraw-Hill Book, 1964.

DASTANE, N.G. Effective rainfall and irrigated water requirements. Rome: FAO, Irrigation and Drainage Paper 25. 1974. 68p.

DOORENBOS, J.; PRUTT, W.O. Guidelines for predicting crop water requirements. Rome: FAO, Irrigation and Drainage Paper 24. 1977. 144p.

FRIZZONE, J.A. Análise de cinco modelos para cálculo de distribuição e frequência de precipitação na região de Viçosa, MG. Viçosa: UFV, 1979. 100p. Dissertação Mestrado

HERSHFIELD, D.M. Effective rainfall and irrigation water requirements. Journal of the Irrigation and Drainage, New York, v.90, p.33-47, 1964

LOUZADA, J.A.; LANNA, A.E.; BELTRAME, L.F.; COTRIN, S.L.; VEZZINI, F.M. Comparação entre modelos diário e mensal de balanço hídrico para estimativa da necessidade de irrigação. In: CONGRESSOBRASILEIRODE IRRIGAÇÃOEDRENAGEM, 9. Anais... Salvador. Brasília: ABID. 1991.p1601-1620.

SAMPAIO, S.C.; CORRÊA, M.M. SOUZA, M.R.; GUIMARÃES, J.C.; SILVA, A.M. Precipitação provável para o Município de Lavras, MG, utilizando a distribuição log-normal. Ciência e Agrotecnologia, Lavras, v.23, n.2, p.382-389, 1999.

SANCHEZ, S.N. Conceptos de lluvia efectiva y su aplicación en la programacion de los riegos. Memorandum Técnico num 307. Secretaria de Recursos Hídráulicos. México. 102p. 1972.

SILVA, W.L.C.; OLIVEIRA, C.A.S.; MORQUELLI, W.A. Subsídios para dimensionamento de sistemas de irrigação. In: CONGRESSO NACIONAL DE IRRIGAÇÃOE DRENAGEM, 8. ABID. Florianópolis, SC, 1988. v.1, p.535-553.

SMITH, M. CROPWAT A computer program for irrigation planning and management. FAO Irrigation and Drainage Paper 46. Rome. 1992. 126p. 